\title{
Transcript profiles of maize embryo sacs and preliminary identification of genes involved in the embryo sac-pollen tube interaction
}

\author{
Shuai Shuai Wang ${ }^{1+}$, Fang Wang ${ }^{2+}$, Su Jian Tan ${ }^{2}$, Ming Xiu Wang ${ }^{2}$, Na Sui ${ }^{3}$ and Xian Sheng Zhang ${ }^{2 *}$ \\ 'College of Animal Science and Veterinary Medicine, Shandong Agricultural University, Tai'an, China \\ 2 State Key Laboratory of Crop Biology, Shandong Key Laboratory of Crop Biology, College of Life Sciences, Shandong Agricultural University, Tai'an, China \\ ${ }^{3}$ College of Life Sciences, Shandong Normal University, Ji'nan, China
}

\author{
Edited by: \\ Tiegang Lu, Chinese Academy of \\ Agricultural Sciences, China \\ Reviewed by: \\ Gabriela Carolina Pagnussat, \\ Universidad Nacional de Mar del \\ Plata, Argentina \\ Tae Kyung Hyun, Gyeongsang \\ National University, South Korea \\ ${ }^{*}$ Correspondence: \\ Xian Sheng Zhang, State Key \\ Laboratory of Crop Biology, \\ Shandong Key Laboratory of Crop \\ Biology, College of Life Sciences, \\ Shandong Agricultural University, \\ Tai'an, Shandong Province, 271018 \\ Shandong, China \\ e-mail: zhangxs@sdau.edu.cn \\ ${ }^{\dagger}$ These authors have contributed \\ equally to this work.
}

The embryo sac, the female gametophyte of flowering plants, plays important roles in the pollination and fertilization process. Maize (Zea mays L.) is a model monocot, but little is known about the interactions between its embryo sac and the pollen tube. In this study, we compared the transcript profiles of mature embryo sacs, mature embryo sacs 14-16 h after pollination, and mature nucelli. Comparing the transcript profiles of the embryo sacs before and after the entry of the pollen tube, we identified 3467 differentially expressed transcripts (3382 differentially expressed genes; DEGs). The DEGs were grouped into 22 functional categories. Among the DEGs, 221 genes were induced upon the entry of the pollen tube, and many of them encoded proteins involved in RNA binding, processing, and transcription, signaling, miscellaneous enzyme family processes, and lipid metabolism processes. Genes in the DEG dataset were grouped into 17 classes in a gene ontology enrichment analysis. The DEGs included many genes encoding proteins involved in protein amino acid phosphorylation and protein ubiquitination, implying that these processes might play important roles in the embryo sac-pollen tube interaction. Additionally, our analyses indicate that the expression of 112 genes encoding cysteine-rich proteins (CRPs) is induced during pollination and fertilization. The CRPs likely regulate pollen tube guidance and embryo sac development. These results provide important information on the genes involved in the embryo sac-pollen tube interaction in maize.

Keywords: transcript profiles, embryo sac, pollen tube, interaction, maize

\section{INTRODUCTION}

Double fertilization involves a complex mechanism in flowering plants. This process begins when the male gametophyte (pollen) produced by anthers reaches the stigma of the pistil. The pollen grain becomes hydrated on the pistil, and germinates a pollen tube that grows directionally through the stigma and style to enter the ovary, if the pollen tube is recognized by female saprophytic tissue. During this process, the pollen tube-pistil interaction allows the pollen tube to grow along the style and transmission tissue, and signals derived from the embryo sac guide the pollen tube into the micropyle (Johnson and Preuss, 2002; Sanchez et al., 2004; Higashiyama and Hamamura, 2008). The embryo sac consists of only a few cells embedded in the ovule tissue of the pistil. Therefore, it is very difficult to analyze the interaction between the pollen tube and the embryo sac.

The embryo sac usually contains four types of cells: the synergids, the egg cell, the central cell, and the antipodal cells. Genome-wide transcriptome analyses have been used to identify genes with potential functions in the embryo sac. To date, genome-wide transcriptome analyses of embryo sacs have been conducted for Arabidopsis, wheat, and maize. The Arabidopsis embryo sac dataset was obtained from cDNA subtraction and microarray analyses of embryo sacs at different developmental stages (Yu et al., 2005). An analysis of a wheat cDNA library of embryo sacs revealed 404 egg-expressed genes (Sprunck et al., 2005). In maize, 3850 embryo-sac-specific transcripts and 963 egg-cell-specific transcripts were detected in embryo sac and egg cell cDNA libraries (Yang et al., 2006). Whole-genome tiling microarray and high-throughput cDNA sequencing analyses identified genes expressed specifically in the ovules of wild-type in Arabidopsis, such as myb98 and dif1 (Jones-Rhoades et al., 2007). Recently, the gene transcript profiles of synergid cells, egg cells, and central cells in the mature Arabidopsis female gametophyte were analyzed via laser-assisted microdissection of individual cells and Affymetrix ATH1 GeneChip expression analyses (Wuest et al., 2010).

The genes identified in embryo sac have been shown to have functions in pollen tube guidance. For example, the synergids have been shown to play a direct role in pollen tube attraction and guidance in the ovules of Arabidopsis, Torenia fournieri, and maize (Higashiyama, 2002; Punwani et al., 2008). The FERONIA (FER) protein is expressed in the synergids, and localizes at the filiform apparatus in Arabidopsis. In fer mutants, wild-type pollen tubes fail to arrest growth and invade the female gametophyte 
without releasing sperms (Huck et al., 2003; Rotman et al., 2003). MYB98 is a transcription factor that is expressed preferentially in the synergid cells of Arabidopsis. The myb98 mutant shows defective organization of the filiform apparatus in synergid cells and defective micropylar guidance of pollen tubes, indicating that the proper function of synergid cells is essential for micropylar guidance (Kasahara et al., 2005). The maize gene ZmEAl is specifically expressed in the egg apparatus. The ZmEA1 protein was shown to attract maize pollen tubes directly in vitro. The pollen tube could not penetrate the intercellular space of the micropyle in ZmEA1-knockdown plants, suggesting that ZmEA1 might be involved in guiding the pollen tube to the micropyle (Dresselhaus and Márton, 2009; Márton and Dresselhaus, 2010). In T. fournieri, LURE1 and LURE2 encode micropylar pollen-tube attractants derived from the synergids (Okuda et al., 2009). As well as the synergids, other female gametophyte cells also function in pollen tube guidance. AtCCG is a putative transcriptional regulator expressed in the central cell. The $c c g$ mutant abolishes micropylar guidance, demonstrating that the central cell is required to guide the pollen tube to the micropyle (Chen et al., 2007). GEX3, a protein localized in the plasma membrane of the male gametophyte and in the egg cell of the female gametophyte, is also essential for pollen tube guidance (Alandete-Saez et al., 2008).

Analyses of the maize genome can provide important information on the molecular mechanism of the embryo sac-pollen tube interaction (Dresselhaus et al., 2011). The quantitative RNAseq analysis of embryo sacs was preformed and used to identify genome features with differential expression between the gametophytes and sporophytic tissues, including protein-coding gene families, duplicated genes and previously unannotated genes (Chettoor et al., 2014). However, the genes involved in postpollination have been never studied at a comprehensive level, and they may be involved in the embryo sac-pollen tube interaction in maize. In this study, the transcriptomes of mature embryo sacs, embryo sacs $14-16 \mathrm{~h}$ after pollination, and mature nucelli of maize were analyzed by RNA-sequencing (RNA-seq). By comparing the transcriptomes of the maize embryo sac before and after entry of the pollen tube, we identified 3467 differentially expressed transcripts (3382 differentially expressed genes; DEGs), including well-known and new genes involved in pollen tube guidance. The results of this study provide new insights into the complex regulatory networks underlying the embryo sac-pollen tube interaction in maize.

\section{MATERIALS AND METHODS ISOLATION OF FEMALE GAMETOPHYTES AND NUCELLI}

The maize inbred line Q319 was used for RNA-seq analyses. The maize plants were cultivated in a field at the Experimental Station of Shandong Agricultural University. Before the experiment, whole ears were covered with paper bags to avoid crosspollination after pollen release. We sampled the ovaries before and after pollination. Mature silks were lightly pollinated with approximately $0.3 \mathrm{~g}$ fresh mature pollen grains at 10:00 AM. Then, $14 \mathrm{~h}$ later, female gametophytes were isolated by enzymatic immersion of ovule slices, as described by Yang et al. (2006). The nucelli were cut directly from the ovule. The female gametophytes and nucelli were frozen in liquid nitrogen and stored at $-80^{\circ} \mathrm{C}$ until RNA extraction.

\section{RNA EXTRACTION AND LIBRARY CONSTRUCTION}

Total RNAs were extracted from embryo sacs and nucelli using an RNeasy Plus Micro kit (Qiagen, Valencia, CA, USA). At least $500 \mathrm{ng}$ total RNA was extracted from each material used for library construction. First, mRNAs extracted from each material were enriched by using oligo(dT) magnetic beads (Illumina, San Diego, CA, USA). The purity and quantity of total RNA was checked (Additional file 1). Then, mRNAs were further enriched by removing rRNAs from the total RNA. The mRNAs were broken into short fragments (approximately $200 \mathrm{bp}$ ) in fragmentation buffer. First-strand cDNA was synthesized with random hexamer primers using the short fragments as templates. Then, dNTPs, buffer, DNA polymerase I, and RNase $\mathrm{H}$ were added to synthesize the second strand. The double-stranded cDNAs were purified with a QiaQuick PCR Extraction kit and washed with EB buffer for end repair and single nucleotide A (adenine) addition. Finally, sequencing adaptors were connected to the fragments. The fragments were purified by agarose gel electrophoresis and enriched by PCR amplification.

\section{ILLUMINA SEQUENCING AND DATA ANALYSIS}

Each library was sequenced using the Illumina HiSeq ${ }^{\mathrm{TM}} 2000$ system at the Beijing Genomics Institute (Shenzhen, China). Single-end 49-bp fragment reads were collected. The sequencing quality of raw data was analyzed by the Illumina Genome Analysis Pipeline, version 1.6. Reads with more than $10 \%$ unknown bases, low-quality raw reads, and reads with adaptors were excluded. The remaining clean reads were used for subsequent analyses. All sequence data have been submitted to the RNA-seq database under accession number GSE57075. The clean reads were aligned to the AGPv2 maize B73 reference genome using the short oligo-nucleotide alignment program v.2 (SOAP2) ( $\mathrm{Li}$ et al., 2009). Mismatches of less than two bases were allowed in this process. According to the alignment, clean reads were divided into unmapped reads, multi-position matched reads, and unique matched reads. For all mapped transcripts with unique matched reads, the original digital gene expression levels were calculated using reads per kilobase per million (RPKM) method (Mortazavi et al., 2008).

We used edgR analysis software (Robinson et al., 2010) to identify preferentially expressed genes in the ES (EPGs) and DEGs before and after pollination. The DEGs (ES vs. Nu, and ESP $v s$. ES) were identified based on a fold change of $\geq 2$ and a false discovery rate (FDR) of $<1 \mathrm{E}-05$. Gene annotations were obtained from the maize B73 sequence AGP V2 5b.60 (http://www.maizesequence. org/index.html).

Genes were classified using MapMan software. The gene ontology (GO) analysis was conducted using the singular enrichment analysis tool (http://bioinfo.cau.edu.cn/agriGO/analysis. php). The sub-cellular location of each gene was predicted using TargetP 1.1. (http://www.cbs.dtu.dk/services/TargetP/). The signal peptide analysis was performed using TargetP 1.1 (http://www.cbs.dtu.dk/services/SignalP//). Protein domains were predicted using the pfam tool (http://pfam.sanger.ac.uk/ 
search?tab=searchSequenceBlock), and the cysteine-rich proteins (CRPs) were predicted and analyzed as described in $\mathrm{Xu}$ et al. (2012).

\section{LASER SCANNING CONFOCAL MICROSCOPY (LSCM) OBSERVATIONS OF MAIZE EMBRYO SAC}

At $14 \mathrm{~h}$ after pollination, mature ovaries were collected from the middle of the ears, immersed in ice-cold FAA fixative, and then placed under vacuum for 20-30 min. Then, the ovaries were immersed in fresh fixative and again placed under vacuum for 20-30 min. The samples were incubated at $4^{\circ} \mathrm{C}$ for $24-48 \mathrm{~h}$, and then whole nucelli were isolated by manual microdissection. The nucelli were rehydrated in a graded ethanol series (v/v: 50, 30, and $10 \%$ ) for $30 \mathrm{~min}$ at each step, and then washed with $100 \%$ distilled water three times for at least $30 \mathrm{~min}$ per wash. Then, the samples were stained with $4 \%(\mathrm{w} / \mathrm{v})$ sucrose red for $12-48 \mathrm{~h}$. The dye was removed from the samples by three washes in $100 \%$ distilled water. The materials were again dehydrated in a graded ethanol series (v/v: 10, 30, 50, 70, 90, and 100\%) for $30 \mathrm{~min}$ at each step, and then finally subjected to three 30 -min washes with $100 \%$ ethanol. The samples were treated with $50 \%$ methyl salicylate (methyl salicylate: ethanol = 1:1) for $2 \mathrm{~h}$, and then with $100 \%$ methyl salicylate for $12-48 \mathrm{~h}$ before observation under a Zeiss LSM-510 confocal microscope (excitation wavelength: $488 \mathrm{~nm}$; filters: BP505-550; Carl Zeiss, Jena, Germany).

\section{IN SITU HYBRIDIZATION}

In situ hybridization was performed as described by Guo et al. (2010). Briefly, the maize ovules were fixed in FAA (v/v: $3.7 \%$ formaldehyde, $5 \%$ acetic acid, $50 \%$ alcohol) overnight at $4^{\circ} \mathrm{C}$. Then, the samples were dehydrated and embedded in paraplast resin (Sigma, St. Louis, MO, USA) and cut into $8-\mu \mathrm{m}$ thick sections. Antisense and sense RNA probes were labeled in vitro from cDNA fragments (448 bp fragments at the $5^{\prime}$ UTR of GRMZM2G165083, 510 bp fragments at the $5^{\prime}$-UTR of GRMZM2G165084) using a digoxigenin RNA labeling kit (Boehringer Mannheim, Mannheim, Germany). They were then hydrolyzed to 200 nucleotides of average length by alkali treatment. After pretreatment, slides were hybridized with the probe (200 ng probe/mL hybridization solution containing 50\% v/v formamide) at $47^{\circ} \mathrm{C}$ overnight. For the detection of hybridized signals, hybridized probes were used with ananti-digoxigenin antibody conjugated with alkaline phosphatase (DIG Nucleic Acid Detection Kit, BoehringerMannheim). Photographs were taken using the Olympus BH-2 microscope.

\section{RT-qPCR ANALYSES}

Total RNA was extracted using the method described above and then treated with RNase-free DNase I (Promega, Madison, WI, USA) to eliminate genomic DNA. Total RNA ( $4 \mu \mathrm{g})$ was used to synthesize cDNA with oligo (dT) primers using M-MLV reverse transcriptase (Promega), according to the manufacturer's instructions. The qRT-PCR analyses were carried out using SYBR Green Real-time PCR Master Mix (Toyobo, Osaka, Japan) with a BioRad CFX96 Real-Time Detection System. For each gene analyzed by qRT-PCR, three biological replicates were analyzed. In each qRT-PCR run, 18S rRNA was used to normalize mRNA levels.
Quantitative variations among different replicates were calculated using the delta-delta threshold cycle relative quantification method. The primers used for qRT-PCR are listed in Additional file 2 .

\section{SEMI-QUANTITATIVE RT-PCR ANALYSES}

Total RNAs were isolated from roots, stems, leaves, pollen grains, and ovaries with TRIzol reagent (Invitrogen, Carlsbad, CA, USA), according to the manufacturer's instructions. The RNAs were treated with DNase I to eliminate genomic DNA (RNase-free; Promega). Details of the primers and cycle conditions are available on request. These experiments were independently replicated at least three times under identical conditions. The transcript level of $18 \mathrm{~s}$ rRNA (detected using the primers $5^{\prime}$-CGGCTACCACATCCAAGGAA- $3^{\prime}$ and $5^{\prime}$ TGTCACTACCTCCCCGTGTCA- $3^{\prime}$ ) was analyzed as the internal control, and was used to normalize all data.

\section{RESULTS AND DISCUSSION TRANSCRIPTOME ANALYSIS OF MAIZE EMBRYO SAC AFTER POLLINATION}

In the maize cultivar Q319, the time from pollination until the arrival of the pollen tube at the embryo sac is approximately $14 \mathrm{~h}$. Therefore, we identified the ES (embryo sac at anthesis) and the ESP (embryo sac at $14 \mathrm{~h}$ after pollination) by laser scanning confocal microscope observation. In the ES, the egg nucleus was present (Figure 1A). In the ESP, the male nucleus was close to the polar nucleus. It suggested that the female and male gametes interact in the ESP (Figure 1B).

To identify the genes involved in the embryo sac-pollen tube interaction, the total RNAs were isolated from the ES and ESP and subjected to RNA-seq analyses. The total RNAs of the nucellus $(\mathrm{Nu})$ at the ES stage was also sequenced as a control. The mRNAs of each of the three tissues were obtained from independent biological samples, and were used to construct cDNA libraries,

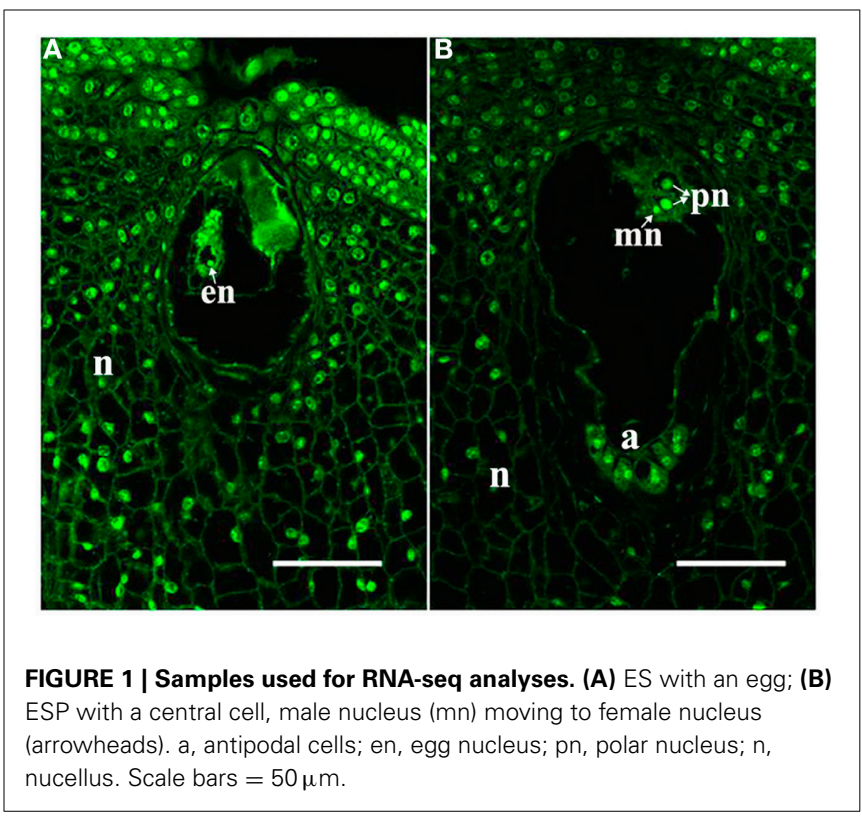


which were then sequenced by the Illumina HiSeq 2000 system. After quality control of sequences (Additional file 3) and removal of 'dirty' raw reads (see Materials and Methods), the number of purity-filtered reads varied from 6,356,811 to 9,049,923 per library (Additional file 3). The tag density was sufficient for quantitative analyses of gene expression. The Spearman's and Pearson's $\mathrm{r}$ values and the slope $(\mathrm{k})$ values were close to 1 , indicating good reproducibility among tissues (Additional file 4).

To identify the genes corresponding to the reads in each library, the filtered clean reads were mapped to version 2 of the maize B73 reference genome (AGPv2, http://www.maizesequence.org) using SOAP2 software. To make the libraries meaningful, transcripts with RPKM values of less than 1 were eliminated before further statistical analyses. Finally, 20,005 transcripts (18,257 genes) in the ES, 22,148 transcripts (19,354 genes) in the $\mathrm{Nu}$, and 17,420 transcripts (16,503 genes) in the ESP were detected (Figure 2, Additional file 5). Among the 20,005 transcripts detected in the ES, approximately $80 \%(15,925 / 20,005)$ were also detected in the ESP. Of them, 809 transcripts ( 803 genes) were specifically expressed in the ESP (Figure 2, Additional file 6). In previous studies, many genes have been identified in cell-specific groups of embryo sac (Lê et al., 2005; Yu et al., 2005; Yang et al., 2006; JonesRhoades et al., 2007; Wuest et al., 2010; Chettoor et al., 2014). Compared with those data, we identified homologs of 176 genes in other species, which were specifically expressed in egg, central cell or synergids (Additional file 6). The information in this study might provide the important gene resources for the network underlying the embryo sac-pollen tube interaction.

\section{CHANGES IN TRANSCRIPT PROFILE OF THE MAIZE EMBRYO SAC AFTER ENTRY OF THE POLLEN TUBE}

In total, 3607 transcripts (3467 genes) were identified as EPGs. These transcripts above 1 RPKM were identified as being differentially expressed in the ES ( $v s$. $\mathrm{Nu}$ ) based on a -fold change

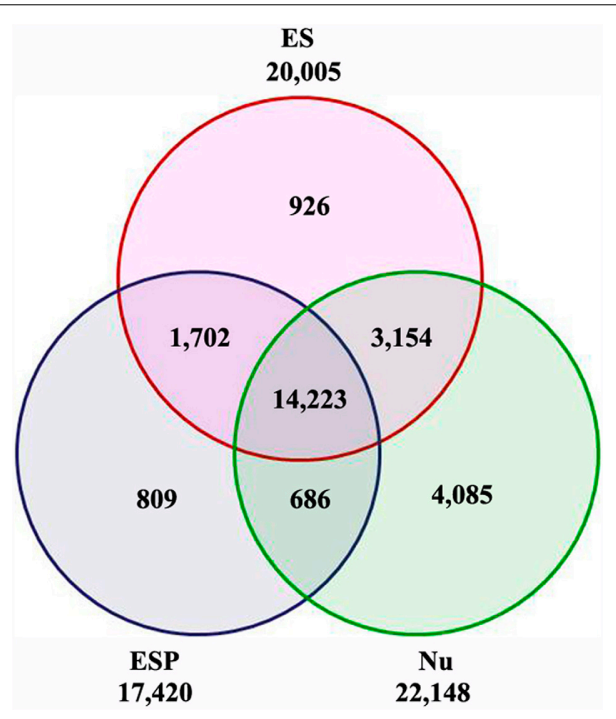

FIGURE 2 | Venn diagram showing number of transcripts detected in ES, ESP, and Nu. of $\geq 2$ and a FDR of $<1 \mathrm{E}-05$ (Additional file 7 ). The quantitative RNA-seq analysis of maize embryo sacs showed that the expression levels of 5011 genes above 0.1 FPKM were 2 fold higher than in ovules with the embryo sacs removed, mature pollen, and seedlings (Chettoor et al., 2014). The 806 genes in EPGs also appeared in the data of Chettoor et al. (2014), suggesting that our data was credible (Additional file 7).

To identify DEGs after entry of the pollen tube, we analyzed digital gene expression using edgR software. The DEGs were identified based on a -fold change of $\geq 2$ and a FDR of $<1 \mathrm{E}-05$. Transcripts with RPKM values of less than 1 were eliminated. In total, 1627 transcripts (1619 genes) were identified as significantly up-regulated and 1840 transcripts (1773 genes) were identified as significantly down-regulated in the ESP, compared with the ES (Additional file 8).

The 3467 DEGs were grouped into 21 functional categories and one unassigned category using MapMan software (Figure 3). The four functional categories with the highest proportions of DEGs were RNA binding, processing and transcription (12.5\%), miscellaneous enzyme family $(6.4 \%)$, signaling transduction $(6.2 \%)$, and protein targeting and degradation (6.1\%). Among the DEGs, $34.5 \%$ were grouped in the "not assigned" category.

Transcription factors (TFs) are a class of proteins that regulate gene transcription and expression by recognizing and binding to cis-acting elements in the promoters of the target genes. Among the 432 genes involved in RNA binding and processing, and transcription, 380 encoded putative TFs. The most abundant TF families were the APETALA2/ethylene-responsive element binding protein (AP2/EREBP), WRKY, chromatin remodeling, homeobox protein $(\mathrm{HB}), \mathrm{v}$-myb avian myeloblastosis viral oncogene homolog (MYB), and mcml-agamous-deficiens serum response factor (MADS) families (Figure 4, Additional file 9). Many members of these subfamilies are known to play important roles in reproductive processes. For example, the AP2/ERF TFs were shown to regulate plant hormone responses (Nakano et al., 2006; Licausi et al., 2010; Sharoni et al., 2011). Both auxin and ethylene regulate ovary and ovule development, and coordinate the development of male and female gametophytes (Zhang and O'Neill, 1993). MYB98, which is specifically expressed in the synergid cells, was shown to have an essential role in pollen tube guidance and in the formation of the filiform apparatus (Kasahara et al., 2005). The MADS-box transcription factor AGL23 was shown to control female gametophyte and embryo development in Arabidopsis (Colombo et al., 2008). In addition, AtCCG, which is expressed in the central cell of the ovule, might act as TF to guide the pollen tube to the micropyle in Arabidopsis (Chen et al., 2007). In our data, the transcript level of the AtCCG-homolog GRMZM2G307720 was significantly lower in the ESP than in the ES. Considering pollination-regulated the expression of TF genes, we suggest that these genes might function in the embryo sac-pollen tube interaction in maize.

In flowering plants, signaling between the pollen tube and the embryo sac is required for fertilization. FER, a putative RLK1-like kinase in Catharanthus roseus (CrRLK1L), is localized at the filiform apparatus, and regulates the male-female interaction during pollen tube reception (Escobar-Restrepo et al., 2007). ANXUR1 and ANXUR2 (ANX1, ANX2), which encode the 


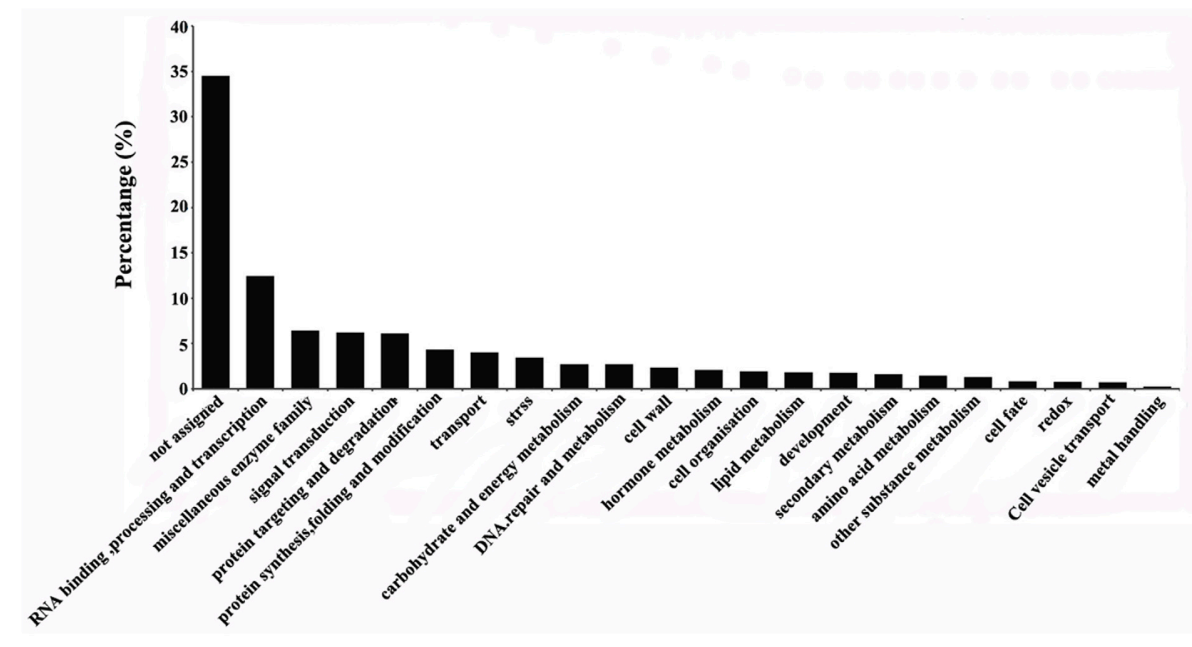

FIGURE 3 | Classification of differentially expressed genes in the ES before and after entry of the pollen tube. Classification results were obtained using MapMan software.

closest homologs of FER-RLK in Arabidopsis, were shown to be preferentially expressed in pollen. The pollen tube of anx1/anx2 mutants ruptured before arriving at the egg apparatus, suggesting that ANX1 and ANX2 might function as male factors controlling pollen tube behavior by directing rupture at the appropriate time (Boisson-Dernier et al., 2009). In our data, there were four transcripts (GRMZM2G100288, GRMZM5G897958, GRMZM2G335052, and GRMZM2G16542) encoding CrRLK1L homologs. Three transcripts (GRMZM2G100288, GRMZM2G335052 and GRMZM2G16542) were up-regulated and one transcript (GRMZM5G897958) was down-regulated after pollination. One of them, GRMZM2G100288, encodes a protein with moderate similarity (approximately 63\%) to FER, suggesting that CrRLK1L homologs might play an important role in the embryo sac-pollen tube interaction in maize.

Interestingly, 221 transcripts were expressed exclusively in the ESP (Additional file 10). We defined these 221 transcripts as those that were induced by the entry of the pollen tube. These genes may encode proteins that function in the pollen-embryo sac interaction in maize. Using MapMan software, the 221 transcripts were grouped into 11 functional categories (Figure 5). As shown in Figure 5, the largest category was un-annotated genes (25\%), followed by genes involved in RNA binding, processing and transcription (12\%), signaling (10\%), miscellaneous enzyme family processes (10\%) and lipid metabolism (6\%).

\section{DIFFERENTIALLY EXPRESSED GENES ENCODING PROTEINS WITH POSSIBLE ROLES IN THE EMBRYO SAC-POLLEN TUBE INTERACTION}

To gain an overall picture of the DEGs, we performed a GO enrichment analysis. The DEGs were grouped into cellular component, molecular function, or biological process categories. The results showed that $17 \mathrm{GO}$ terms were overrepresented among the DEGs, based on a $p$ value of $<0.001$ and an FDR of $\leq 0.05$ (Table 1). Within the biological process category, there were many DEGs in the response to stress, protein amino acid phosphorylation, and protein ubiquitination subcategories. This result indicated that these pathways and processes might be important in the embryo sac-pollen tube interaction.

\section{PROTEIN UBIQUITINATION}

The ubiquitin-proteasome system (UPS) consists of five components: ubiquitin, ubiquitin activating enzyme E1, ubiquitin-conjugating enzyme E2, ubiquitin ligase enzyme E3, and proteasomes. The UPS system appears to play a central role in spermatogenesis and fertilization (Nakamura, 2013). Approximately 70 E3 ubiquitin ligases are expressed during spermatogenesis in mice (Hou et al., 2012). In mammals, abnormal spermatozoa are marked with ubiquitin during epididymal passage. Subsequently, most of the ubiquitinated spermatozoa are phagocytosed by the epididymal epithelial cells, indicating that ubiquitin mediates sperm quality control and regulates male fertility (Sutovsky et al., 2001). In flowering plants, there are relatively high levels of protein ubiquitination in generative cells and sperm cells, suggesting that the UPS may be involved in the development of the male gametophyte (Singhmb et al., 2002). The small ubiquitin-like modifier (SUMO) E3 ligase, SAP, and Miz-finger domain-containing protein 1 (SIZ1) maintain the stability and normal function of the mature female gametophyte, which is necessary for pollen tube guidance in Arabidopsis (Ling et al., 2012). Previously, we showed that UPS proteins, especially E3 ubiquitin ligases, are involved in the pollen tube-silk interaction in maize (Xu et al., 2013).

The GO analysis revealed that 19 genes related to protein ubiquitination were among the DEGs (Table 1). A further MapMan analysis showed that 17 of these 19 genes encode Ring-type E3 ubiquitin ligases (Additional file 11). These findings suggest that UPS proteins, such as E3 ubiquitin ligases, are likely to be involved in the development of the embryo sac and in the embryo sac-pollen tube interaction in maize.

\section{PROTEIN AMINO ACID PHOSPHORYLATION}

Protein amino acid phosphorylation is involved in almost all cellular processes. GO enrichment analysis of the DEGs showed 


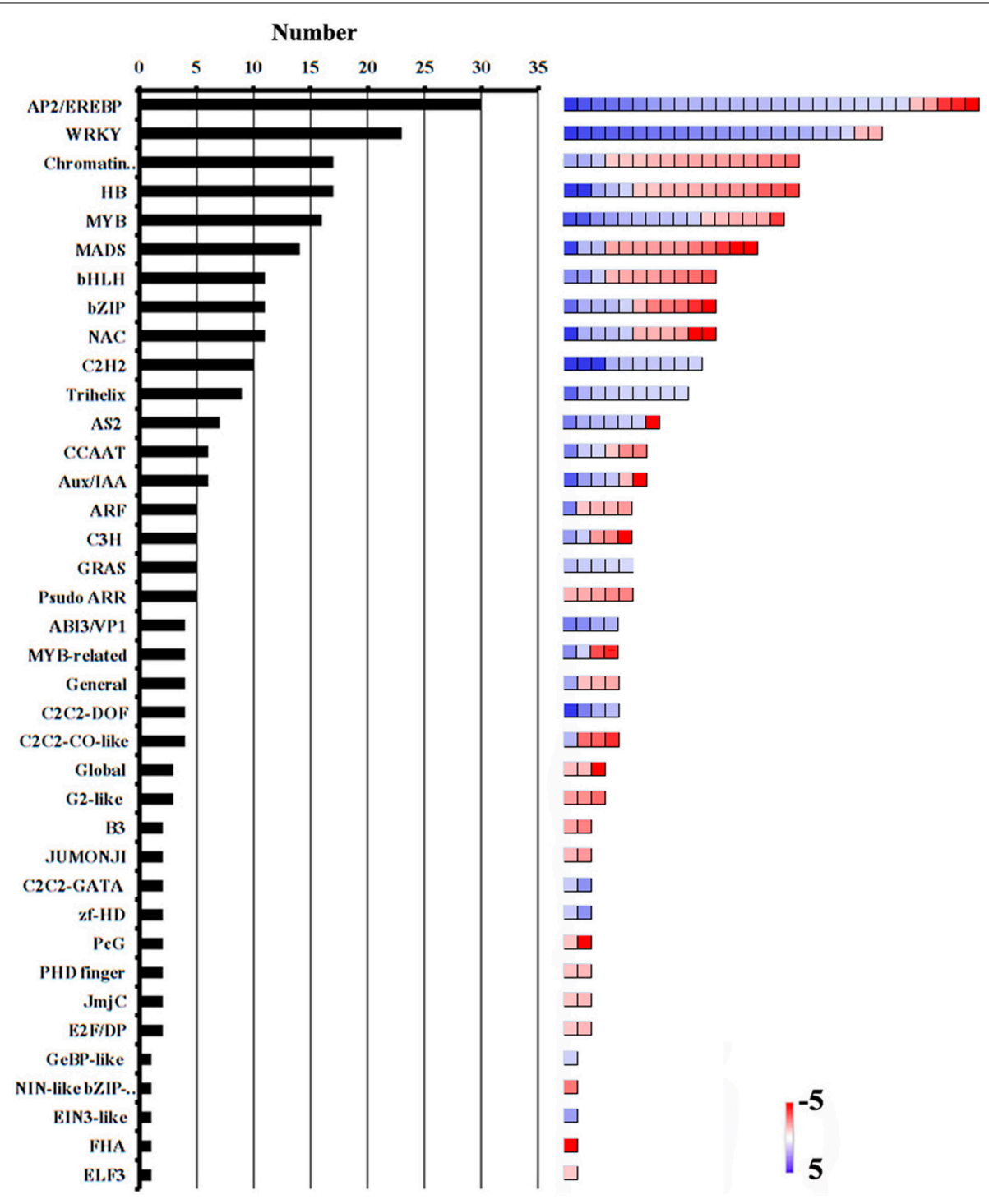

FIGURE 4 | Abundance of TF families among DEGs. Number of genes in each category is shown on the left. Color scales (right) show relative transcript abundance of each gene ( $\log _{2}$-transformed RPKM values) in the given categories.

that there were 172 genes related to protein amino acid phosphorylation (Table 1, Additional file12), suggesting that many proteins in the embryo sac were phosphorylated after the pollen tube had entered the embryo sac. Interestingly, we found that genes related to protein amino acid phosphorylation were enriched in $\mathrm{Nu}$, but not in ES. This finding suggests that there was a lower level of protein phosphorylation in the mature embryo sac than in the sporophyte. Thus, it is likely that protein phosphorylation events are involved in the embryo sac-pollen tube interaction.

The 172 genes involved in protein amino acid phosphorylation were further classified using MapMan software (Figure 6, Additional file 12). This analysis showed that the gene terms related to post-translational modification (26.1\%), LRR-XI RLK (13.4\%), DUF26 RLK (12.2\%), RLCK VII (7.5\%) and LRRIII RLK (5.2\%), were overrepresented. Furthermore, 113 genes encoding receptor-like kinases (RLKs) were identified. These genes were further classified into 22 categories (Figure 6). The largest category consisted of genes encoding LRR-XI RLKs. These kinases are involved in various aspects of meristem development and nodulation (Brand et al., 2000; Krusell et al., 2002; Schnabel et al., 2005). Arabidopsis PEPR1 is a typical LRR receptor kinase with an extracellular LRR domain and an intracellular protein kinase domain. It belongs to the LRR-XI subfamily (Shiu et al., 2004). PEPR2 shares 76\% amino acid similarity with PEPR1 (Yamaguchi et al., 2006). Both PERP1 and PERP2 could recognize damage associated with molecular patterns and active innate immunity pathways (Yamaguchi et al., 2006, 2010; Krol et al., 2010). Previous study proposed that conserved molecular components are involved in both pollen tube reception and fungal invasion in Arabidopsis (Kessler et al., 2010). In our data, the expression levels of 24 LRR-XI RLKs were significantly altered before and after pollination (Additional file 12). One of them, 


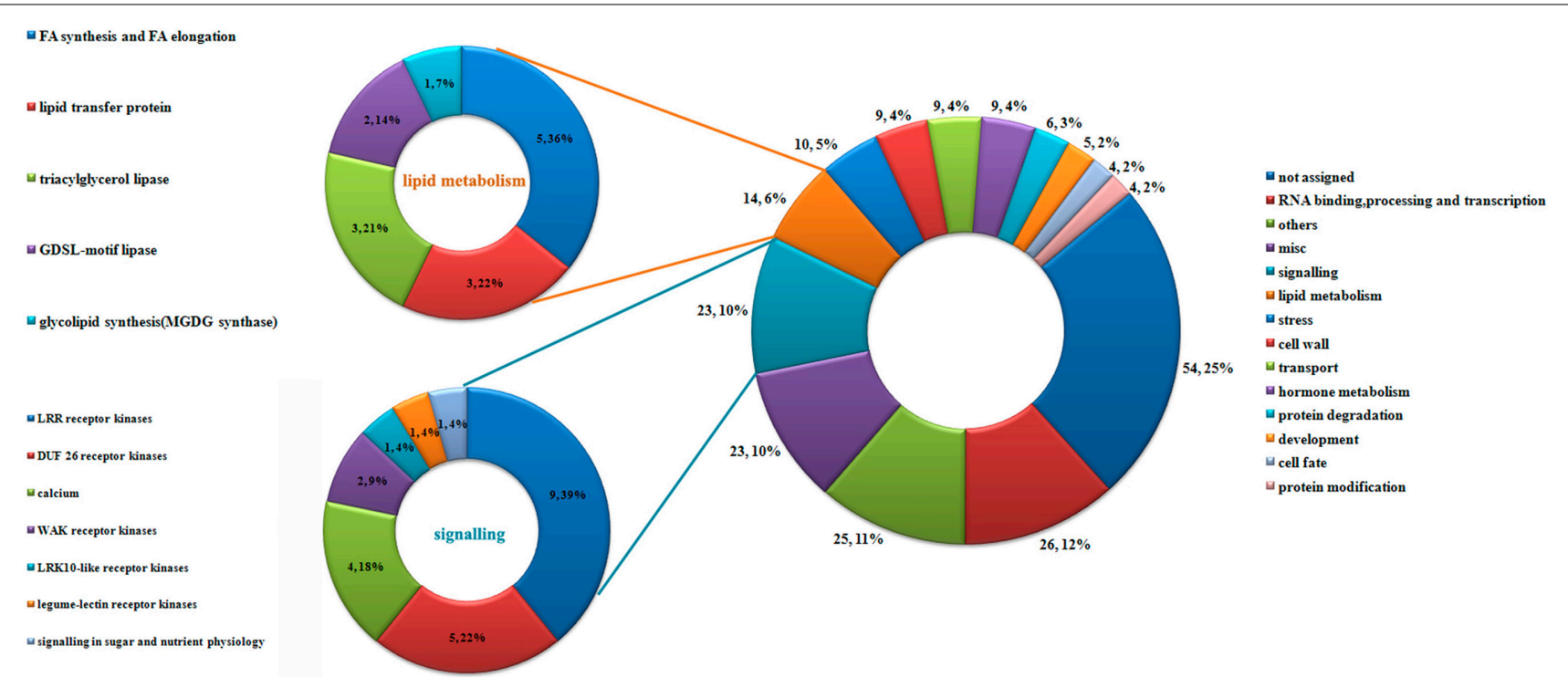

FIGURE 5 | Classification of genes induced by pollination. Using MapMan, 221 genes induced by pollination were classified into 14 categories; details of genes involved in lipid metabolism and signaling are shown on the left.

Table 1 | Over-represented functional GO terms among DEGs in the maize ES before and after entry of the pollen tube.

\begin{tabular}{|c|c|c|c|c|c|c|c|}
\hline GO Term ${ }^{a}$ & Term type $^{a}$ & Query item ${ }^{b}$ & Query total ${ }^{c}$ & $\mathrm{Bg}_{\text {item }}^{\mathrm{d}}$ & Bg total ${ }^{e}$ & $p$-Value ${ }^{f}$ & FDR $^{\mathrm{g}}$ \\
\hline Negative regulation of molecular function & $P$ & 13 & 2068 & 66 & 39203 & 3.70E-05 & 0.012 \\
\hline L-phenylalanine metabolic process & $P$ & 9 & 2068 & 34 & 39203 & 5.00E-05 & 0.013 \\
\hline Response to stress & $P$ & 214 & 2068 & 3059 & 39203 & $5.40 \mathrm{E}-05$ & 0.013 \\
\hline Protein ubiquitination & $P$ & 19 & 2068 & 139 & 39203 & 0.00014 & 0.026 \\
\hline $\begin{array}{l}\text { Hydrolase activity, acting on glycosyl } \\
\text { bonds }\end{array}$ & $\mathrm{F}$ & 74 & 2068 & 821 & 39203 & 9.60E-06 & 0.003 \\
\hline Ammonia-lyase activity & $\mathrm{F}$ & 8 & 2068 & 24 & 39203 & $2.10 \mathrm{E}-05$ & 0.0049 \\
\hline Protein serine/threonine kinase activity & $\mathrm{F}$ & 165 & 2068 & 2248 & 39203 & 3.30E-05 & 0.006 \\
\hline ATP binding & $\mathrm{F}$ & 341 & 2068 & 5133 & 39203 & 4.30E-05 & 0.0061 \\
\hline $\begin{array}{l}\text { Transferase activity, transferring acyl } \\
\text { groups other than amino-acyl groups }\end{array}$ & $\mathrm{F}$ & 36 & 2068 & 357 & 39203 & 0.0002 & 0.017 \\
\hline Identical protein binding & $\mathrm{F}$ & 13 & 2068 & 79 & 39203 & 0.00025 & 0.019 \\
\hline Copper ion binding & $\mathrm{F}$ & 22 & 2068 & 194 & 39203 & 0.00067 & 0.046 \\
\hline
\end{tabular}

${ }^{a}$ GO term classifications: P, biological process; $C$, cellular component; F, molecular function.

${ }^{b}$ Query item number in ES preferentially expressed genes.

${ }^{c}$ Total annotated query item number in agriGO.

${ }^{d}$ Query item number in maize genome version $5 a$.

e Total annotated item number in maize genome version $5 a$.

${ }^{f}$ Determined by Fisher's exact test.

${ }^{g}$ Determined by the Benjamini-Hochberg-Yekutieli procedure.

GO terms with $p$-value $<0.001$ and FDR $\leq 0.05$ were regarded as over-represented terms. 


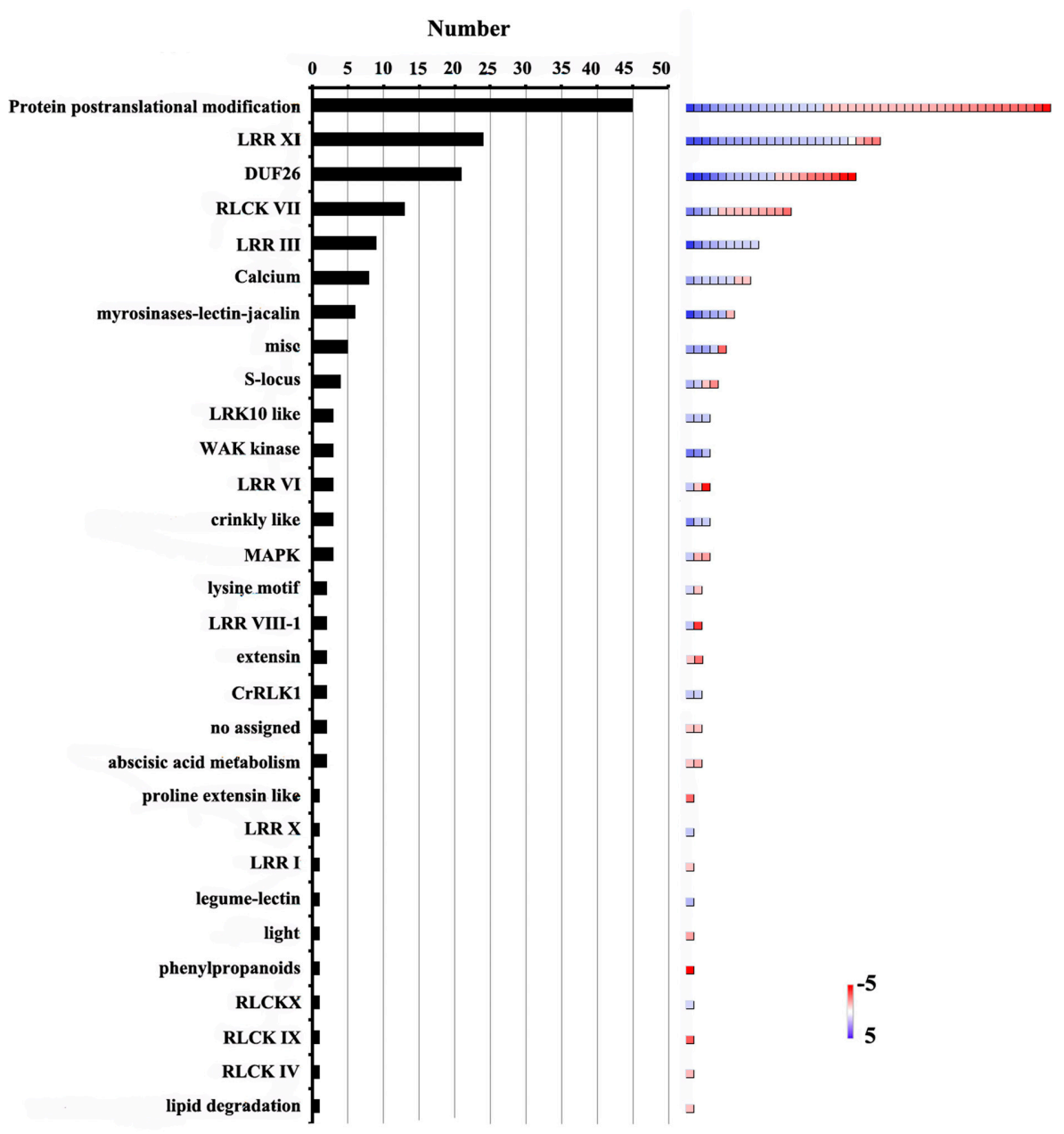

FIGURE 6 | Distribution of genes involved in protein amino acid phosphorylation among DEGs. Number of genes in each category is shown on the left. Color scales (right) show relative transcript abundance of each gene ( $\log _{2}$-transformed RPKM values) in the given categories.

GRMZM2G428554 was homologous to PEPR1, implying that LRR-XI RKLs might be involved in the interaction between the embryo sac and the pollen tube in maize.

\section{STRESS RESPONSES}

It was suggested that there were similarities in genetic programs controlling pollination/fertilization and stress responses in rice (Lan et al., 2005). Here, totally we identified 214 genes related to stress responses in DEGs by GO analysis (Table 1, Additional 13). Of them, sixty-two genes were down-regulated and 152 genes were up-regulated, respectively. The 214 genes involved in stress responses were further classified using MapMan software (Figure 7). Most genes are involved in peroxidases, transcription factors, and signaling and cell wall. Stigmas have long been known to exhibit high levels of peroxidase activity when receptive to pollen (Dupuis and Dumas, 1990; Dafni and Motte Maues, 1998; McInnis et al., 2005). The Stigma-Specific Peroxidase (SSP) protein was identified and characterized in Senecio squalidus (McInnis et al., 2005). Senecio stigmas accumulate high levels of ROS in the epidermal cells (papillae) where SSP is localized
(McInnis et al., 2006). In Arabidopsis, FER give rise to a high ROS environment at the entrance of the female gametophyte and it can mediate pollen tube rupture to release sperms for fertilization (Duan et al., 2014). In DEGs, GRMZM2G100288 was homologous to FER, and there were 13 genes encoding the peroxidases, implying that ROS levels of embryo sac may have been changed after pollination (Additional file 13). Thus, the genetic network of stress response might also function in pollination in maize.

\section{CYSTEINE-RICH PEPTIDES MAY REGULATE POLLEN TUBE GUIDANCE AND EMBRYO SAC DEVELOPMENT}

In plants, small secreted peptides (SSPs) play critical roles in defense, development, and many other physiological processes. Recent studies have revealed that various SSPs are involved in the pollen-pistil interaction. Interestingly, many of them are CRPs (Higashiyama, 2010; Chae and Lord, 2011). The maize CRP $\mathrm{ZmES} 4$ was shown to mediate pollen tube rupture by regulating the activity of the potassium channel protein KZM1 (Amien et al., 2010). Therefore, we speculated that CRPs may be involved in pollen tube guidance in maize. To test this hypothesis, we 


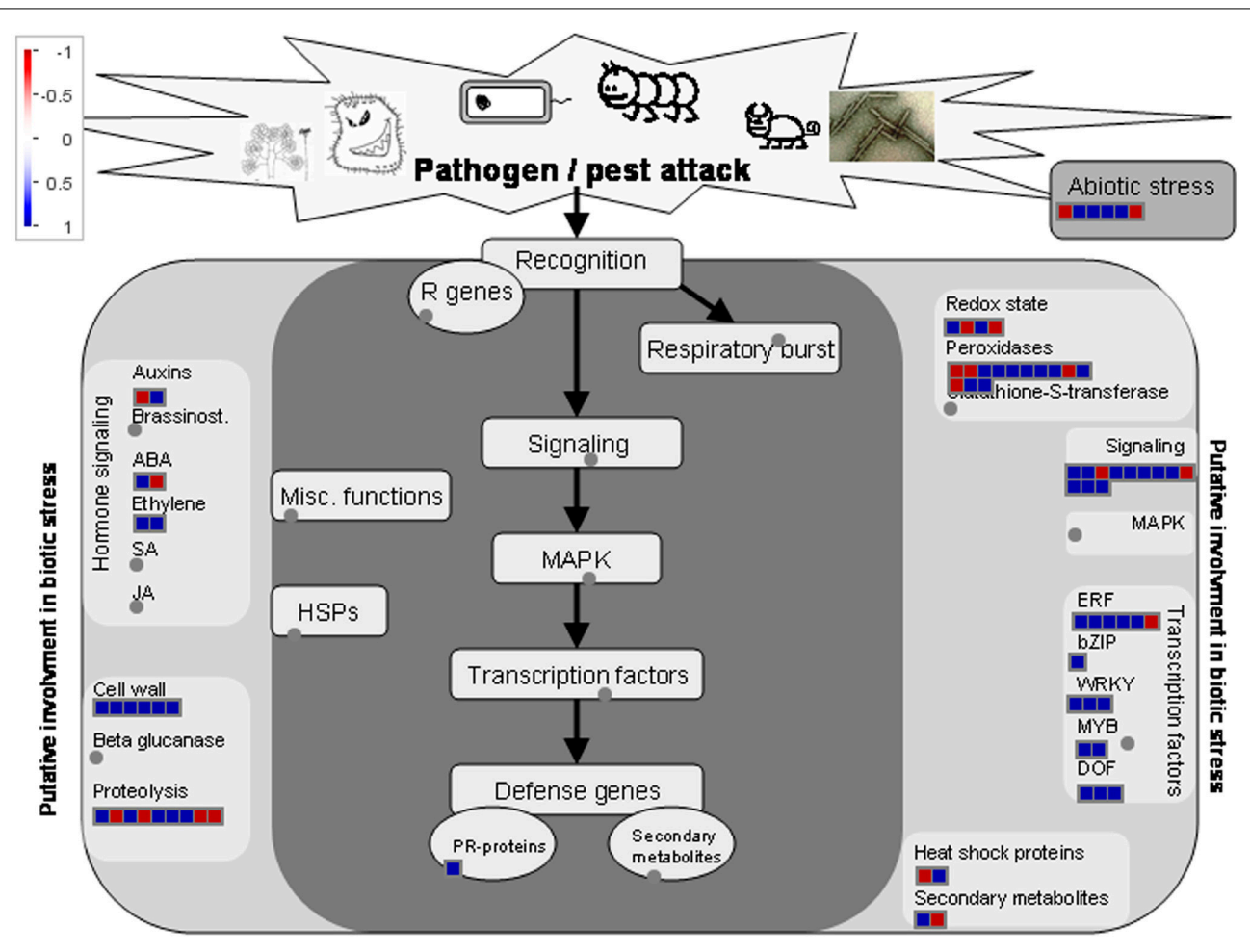

FIGURE 7 | Transcripts involved in stress responses. Transcript expression levels represented by $\log _{2}$ transformed RPKM values of ESP relative to $E S$, The color of each box represents the level of up/down regulation of each transcript. Red represents down-regulation, blue represents up-regulation. The number of transcripts in each gene family is equal to the number of boxes in each gene family. The relative expression is represented by color scales as indicated. screened all the predicted proteins encoded by DEGs to identify proteins with a molecular weight of $20 \mathrm{kD}$ or less, and identified 139 small proteins. Of them, 57 CRPs preferentially expressed in the ES ( $v s$. ESP) were identified by local BLAST searches, using the cysteine arrangement model reported by Silverstein et al. (2007) as the search query. Using the same strategy, we identified 87 CRPs that were preferentially expressed in the ES (vs. $\mathrm{Nu}$ ), and among them, 32 CRPs were preferentially expressed in the ES (vs. ESP) (Table 2). In total, 112 CRPs were identified among DEGs and EPGs.

CRPs can be grouped into classes according to their domains. The classes of the 112 CRPs were identified based on their conserved domains, using tools at the Conserved Domain database (http://pfam.sanger.ac.uk/search? $\mathrm{tab}=$ searchSequenceBlock) (Table 2 ). In previous studies, four maize CRPs containing the gamma-thionin domain (ZmES1, 2,3 , and 4) expressed in the embryo sac were shown to be involved in the embryo sac-pollen tube interaction (Cordts et al., 2001; Amien et al., 2010). In our dataset, GRMZM2G012012, GRMZM2G359046, GRMZM2G128301, and GRMZM2G009359 were identified as ZmES1, 2, 3, and 4, respectively, by BLAST sequence analysis. Pollen-specific rapid alkalinization factors (RALFs) function in pollen tube elongation (Covery et al., 2010). In our data set, three genes encoding maize RALFs (GRMZM2G317406, GRMZM2G148064, and GRMZM173747) were preferentially expressed in the ES. In lily, the stigma/stylar cysteine-rich adhesion (SCA) protein, a lipid-transfer protein containing a Tryp-alpha-amyl domain, was shown to be abundant in the stigma and the transmitting tract, and was responsible for pollen tube growth and guidance. Chemocyanin, belonging to the blue copper domain-containing protein family, was shown to induce pollen tube chemotropism, and its activity was enhanced by SCA (Kim et al., 2003, 2006; Lord, 2003; Park and Lord, 2003). In this study, there was one SCA homolog (GRMZM2G101958) among the DEGs. Among the EPGs, there were four genes (GRMZM2G023847, GRMZM2G0146015, GRMZM2G0004160, and GRMZM2G1128531) encoding products with a copper-binding protein-like (cu_bind_like) domain. One of them encoded a protein (GRMZM2G004160) showing $61.5 \%$ similarity to chemocyanin. Interestingly, the transcript level of GRMZM2G0004160 differed markedly before and after the entry of the pollen tube. These results suggested that the interaction between SCA and chemocyanin might play an important role during pollination in maize.

The Cys-rich late anther tomato 52 protein (encoded by LAT52) was shown to be essential for pollen hydration and pollen tube growth. The LAT52 protein in tomato is an Ole e 1-like (Pollen Ole e I) domain-containing protein with a pollen-specific expression pattern (Muschietti et al., 1994; Jiang et al., 2005). Among the EPGs there were three genes (GRMZM2G317406, GRMZM2G148064, and GRMZM173747) encoding proteins with a Pollen Ole e I domain. One of them, 
Table 2 | Distribution of CRPs among DEGs and EPGs.

\begin{tabular}{|c|c|c|c|c|c|}
\hline GENEID & Domain & Pattern $\log _{2}(E S P / E S)^{a}$ & FDR & Pattern $\log _{2}(E S / N u)^{b}$ & FDR \\
\hline GRMZM2G101584_T01 & Gamma-thionin & -2.36 & $2.44 \mathrm{E}-74$ & 10.84 & 0 \\
\hline GRMZM2G047842_T01 & Gamma-thionin & & & 9.77 & 3.00E-115 \\
\hline GRMZM2G125520_T01 & Gamma-thionin & -1.66 & $5.61 \mathrm{E}-42$ & 11.65 & 0 \\
\hline GRMZM2G359064_T01 & Gamma-thionin & & & 32.76 & $5.92 \mathrm{E}-10$ \\
\hline GRMZM2G128301_T01 & Gamma-thionin & & & 36.08 & 5.86E-79 \\
\hline GRMZM2G009359_T01 & Gamma-thionin & & & 38.87 & $3.30 \mathrm{E}-248$ \\
\hline GRMZM2G357124_T01 & RALF & & & 32.62 & 3.77E-09 \\
\hline GRMZM2G383303_T01 & RALF & -1.77 & $2.70 \mathrm{E}-19$ & 36.59 & 3.23E-102 \\
\hline GRMZM2G077259_T01 & RALF & 1.1 & 8.17E-07 & 1.57 & 5.28E-09 \\
\hline GRMZM2G301663_T01 & RALF & 1.06 & 1.17E-10 & & \\
\hline GRMZM2G165083_T01 & Toxin_3 & 2.03 & 1.43E-15 & 33.54 & $5.15 \mathrm{E}-17$ \\
\hline GRMZM2G097084_T01 & Toxin_3 & & & 36.35 & 8.90E-91 \\
\hline AC209356.4_FGTO01 & Toxin_3 & 2.18 & 1.42E-12 & 32.81 & 1.74E-10 \\
\hline AC199577.4_FGTO04 & Toxin_3 & & & 38.29 & 1.03E-205 \\
\hline GRMZM2G045082_T01 & Toxin_3 & & & 8.84 & 7.73E-180 \\
\hline GRMZM2G171597_T01 & LTP_2 & 1.57 & $1.59 \mathrm{E}-13$ & 7.42 & 1.77E-31 \\
\hline GRMZM2G084325_T01 & LTP_2 & & & 8 & $7.25 E-45$ \\
\hline GRMZM2G071575_T01 & LTP_2 & & & 3.97 & 1.44E-94 \\
\hline GRMZM2G089400_T01 & LTP_2 & 1.48 & $9.88 \mathrm{E}-16$ & & \\
\hline AC225127.3_FGT003 & Tryp_alpha_amyl & & & 5.38 & 4.46E-08 \\
\hline GRMZM2G404688_T01 & Tryp_alpha_amyl & & & 32.06 & $1.90 \mathrm{E}-06$ \\
\hline GRMZM2G101958_T01 & Tryp_alpha_amyl & & & 2.94 & $2.41 \mathrm{E}-83$ \\
\hline GRMZM2G406313_T01 & Tryp_alpha_amyl & 32.18 & $2.49 \mathrm{E}-06$ & & \\
\hline GRMZM2G010868_T01 & Tryp_alpha_amyl & 6.29 & $5.38 \mathrm{E}-33$ & & \\
\hline GRMZM5G898755_T01 & Tryp_alpha_amyl & -2.18 & 8.79E-09 & & \\
\hline GRMZM2G023847_T01 & Cu_bind_like & -3.95 & $5.24 \mathrm{E}-56$ & 9.7 & 1.68E-111 \\
\hline GRMZM2G146015_T01 & Cu_bind_like & 1.04 & 7.93E-14 & 1.2 & $2.33 \mathrm{E}-15$ \\
\hline GRMZM2G004160_T01 & Cu_bind_like & -1.89 & 2.67E-05 & 2.54 & $1.50 \mathrm{E}-11$ \\
\hline GRMZM2G128531_T01 & Cu_bind_like & & & 1.98 & $1.73 \mathrm{E}-23$ \\
\hline GRMZM2G027198_T01 & Cu_bind_like & 1.22 & 2.37E-23 & & \\
\hline GRMZM2G317406_T01 & Pollen_Ole_e_I & & & 32.67 & 2.04E-09 \\
\hline GRMZM2G148064_T01 & Pollen_Ole_e_l & & & 32.33 & $1.55 \mathrm{E}-07$ \\
\hline GRMZM2G173747_T01 & Pollen_Ole_e_l & & & 2.08 & 2.14E-48 \\
\hline GRMZM2G022347_T01 & Pollen_Ole_e_I & 3.44 & $6.24 \mathrm{E}-07$ & & \\
\hline GRMZM2G068202_T01 & GASA & 1.39 & $2.80 \mathrm{E}-09$ & 7.19 & 2.59E-27 \\
\hline GRMZM2G172596_T01 & GASA & -2.64 & $2.21 \mathrm{E}-46$ & 2.39 & 2.94E-49 \\
\hline GRMZM2G117940_T01 & GASA & 2.66 & $3.74 \mathrm{E}-43$ & 7.29 & 3.50E-29 \\
\hline GRMZM2G105364_T01 & GASA & 2.3 & $2.35 \mathrm{E}-58$ & & \\
\hline
\end{tabular}


Table 2 | Continued

\begin{tabular}{|c|c|c|c|c|c|}
\hline GENEID & Domain & Pattern $\log _{2}(E S P / E S)^{a}$ & FDR & Pattern $\log _{2}(E S / N u)^{b}$ & FDR \\
\hline GRMZM2G149869_T01 & SLR1-BP & -1.14 & $2.39 E-16$ & 38.56 & $3.92 E-225$ \\
\hline GRMZM2G042125_T01 & Prolamin_like & & & 10.88 & 1.39E-254 \\
\hline GRMZM2G083190_T01 & Prolamin_like & & & 9.19 & 3.80E-204 \\
\hline GRMZM2G119812_T01 & Stig1 & 1.17 & $1.30 \mathrm{E}-06$ & 7.21 & $8.75 E-28$ \\
\hline GRMZM2G041039_T01 & AmiS_Urel & 1.38 & $6.43 \mathrm{E}-14$ & 5.37 & 7.10E-50 \\
\hline GRMZM2G146573_T01 & DUF1180 & & & 7.63 & $6.23 E-88$ \\
\hline GRMZM2G129083_T01 & DUF1180 & & & 1.18 & 7.05E-14 \\
\hline GRMZM2G079499_T01 & DUF3742 & & & 33.06 & $2.41 \mathrm{E}-12$ \\
\hline GRMZM2G050994_T01 & DUF2667 & & & 11.38 & 0 \\
\hline GRMZM2G175165_T01 & DUF2667 & -2 & $4.86 \mathrm{E}-55$ & 13.08 & 0 \\
\hline GRMZM2G021621_T01 & DPBB_1 & 1.95 & 1.47E-25 & & \\
\hline GRMZM2G150172_T01 & NO & & & 2.53 & 1.95E-66 \\
\hline GRMZM2G014994_T01 & NO & & & 2.33 & $2.00 \mathrm{E}-28$ \\
\hline GRMZM2G121256_T01 & NO & & & 33.48 & 1.67E-16 \\
\hline GRMZM2G136534_T03 & NO & & & 2.82 & $6.69 \mathrm{E}-07$ \\
\hline AC212323.4_FGTOO5 & NO & 1.16 & $2.12 \mathrm{E}-10$ & 35.44 & $1.91 \mathrm{E}-55$ \\
\hline GRMZM2G044174_T01 & NO & & & 2.32 & 4.07E-08 \\
\hline GRMZM2G019644_T01 & NO & & & 38.11 & 1.30E-193 \\
\hline GRMZM2G112855_T01 & NO & -1.98 & 4.62E-41 & 38.46 & 4.03E-218 \\
\hline GRMZM2G046252_T01 & NO & 1.4 & $6.37 \mathrm{E}-21$ & 36.5 & $1.24 \mathrm{E}-97$ \\
\hline GRMZM2G463167_T01 & NO & 1.45 & $5.66 \mathrm{E}-20$ & 36.05 & $1.04 \mathrm{E}-77$ \\
\hline GRMZM2G088169_T01 & NO & 2.12 & $2.15 E-36$ & 8.24 & $7.95 E-52$ \\
\hline GRMZM2G040020_T01 & NO & & & 34.3 & $8.75 E-28$ \\
\hline GRMZM2G040020_T02 & NO & & & 33.24 & $6.42 \mathrm{E}-14$ \\
\hline GRMZM2G006601_T01 & NO & -1.44 & 7.55E-33 & 12.3 & 0 \\
\hline GRMZM5G878322_T01 & NO & 1.2 & $2.92 \mathrm{E}-07$ & 2.11 & 4.17E-12 \\
\hline GRMZM2G145466_T01 & NO & -2.34 & 1.75E-68 & 39.9 & 0 \\
\hline GRMZM2G483273_T01 & NO & & & 11.97 & 0 \\
\hline GRMZM2G489599_T01 & NO & & & 11.72 & 0 \\
\hline GRMZM2G074293_T01 & NO & & & 11.99 & 0 \\
\hline GRMZM2G483275_T01 & NO & & & 10.5 & 0 \\
\hline GRMZM2G489627_T01 & NO & & & 9.48 & 1.05E-224 \\
\hline GRMZM2G167151_T01 & NO & & & 13.21 & 0 \\
\hline GRMZM2G029926_T01 & NO & & & 31.98 & $6.58 \mathrm{E}-06$ \\
\hline GRMZM2G079962_T01 & NO & -3.16 & $8.28 \mathrm{E}-140$ & 12.35 & 0 \\
\hline GRMZM2G055629_T01 & NO & -2.31 & 4.85E-11 & 7.27 & $5.17 \mathrm{E}-106$ \\
\hline GRMZM2G055629_T02 & NO & & & 34.73 & $2.11 \mathrm{E}-36$ \\
\hline GRMZM2G180903_T02 & NO & -4.81 & $3.35 \mathrm{E}-07$ & & \\
\hline GRMZM2G346499_T01 & NO & 1.18 & 1.97E-12 & & \\
\hline GRMZM2G439337_T01 & NO & -1.68 & 3.06E-05 & & \\
\hline GRMZM2G166094_T01 & NO & 1.12 & $9.51 \mathrm{E}-07$ & & \\
\hline AC211652.4_FGTO01 & NO & 1.38 & 3.76E-07 & & \\
\hline GRMZM2G150688_T01 & NO & -2.78 & $1.64 \mathrm{E}-07$ & & \\
\hline
\end{tabular}

${ }^{a}$ Gene expression levels ( $\log _{2}$-transformed RPKM values in ESP relative to ES).

${ }^{b}$ Gene expression levels ( $\log _{2}$-transformed RPKM values in ES relative to $\mathrm{Nu}$ ). 
GRMZM2G148064, was homologous to LAT52. A gene encoding a LAT52 homolog (GRMZM022347) was also present in the ESP/ES DEGs dataset. Two small secreted Brassica pollen coat proteins, SLR1-BP1 and SLR1-BP2, were shown to interact with SLR1 during pollen grain adhesion (Luu et al., 1999; Takayama et al., 2000). GRMZM0149869 (corresponding to SLR1-BP1) and GRMZM00977 were present in the EPGs dataset; the transcript level of GRMZM0149869 was lower in the ESP than in the ES.

Animal toxins have been shown to modulate $\mathrm{K}^{+}$channels, $\mathrm{Na}^{+}$channels, or $\mathrm{Ca}^{2+}$-activated $\mathrm{K}^{+}$channels either as pore blockers or as gating modifiers (Mouhat et al., 2004). Until now, there are a few studies on the CRPs with a Scorpion toxin-like domain (Toxin_3 domain) in plants. AsG255 was expressed abundantly in nodules in Astragalus sinicus, and its protein was identified to contain a scorpion toxin-like domain at the C-terminus, which might function as the common signaling component involved in the plants' perception of soil microorganisms (Chou et al., 2006). Five genes encoding CRPs with toxin domains were present in the EPGs dataset (GRMZM2G045082, GRMZM2G165083, GRMZM2G097084, AC199577.4, and AC209356.4). All of these genes encoded proteins with a Toxin_3 domain. Two genes (GRMZM2G165083 and GRMZM2G097084) were selected for in situ hybridization analysis in mature embryo sacs. The GRMZM2G165083 mRNA accumulated in the egg cell, central cell, and antipodal cells (Figures 8A-C). GRMZM2G097084 transcripts were detected in the embryo sac, and also in the integuments around the micropyle (Figures 8D-F). Semiquantitative PCR analyses confirmed that the two genes were preferentially or specifically expressed in ovaries (Figure 8G). Further analysis showed the transcript levels of GRMZM2G165083 and AC209356.4 were higher in the ESP than in the ES in DEGs (Table 2). Together, these results suggested that toxin peptides might function in embryo sac development and/or in pollen tube guidance in maize.

\section{VALIDATION OF RNA-Seq RESULTS}

To validate the RNA-seq analysis, ten genes were selected randomly for real-time qRT-PCR analyses. The details of these transcripts and their specific primers are shown in Additional file 2. The transcript profiles obtained by RT-qPCR were strongly correlated with those obtained in the RNA-seq analysis $(R=0.9495)$, confirming the reliability of the RNA-seq data (Figure 9).

\section{CONCLUSIONS AND PERSPECTIVES}

In maize, the journey of the pollen tube toward the embryo sac can be divided into five phases. In phase I-III, the pollen grains adhere, hydrate and germinate on stigma cells. In phase IV, the pollen tubes grow between the sporophytic cell layers. In phase $\mathrm{V}$, pollen tubes leave the sporophytic tissue and enter the ovary cavity (Heslop-Harrison et al., 1985; Johnson and Preuss, 2002; Lausser and Dresselhaus, 2010). During phases I-IV, the pollen tubes are controlled by signals from the sporophyte, however in phase $\mathrm{V}$, they are controlled by signals from the gametophyte.

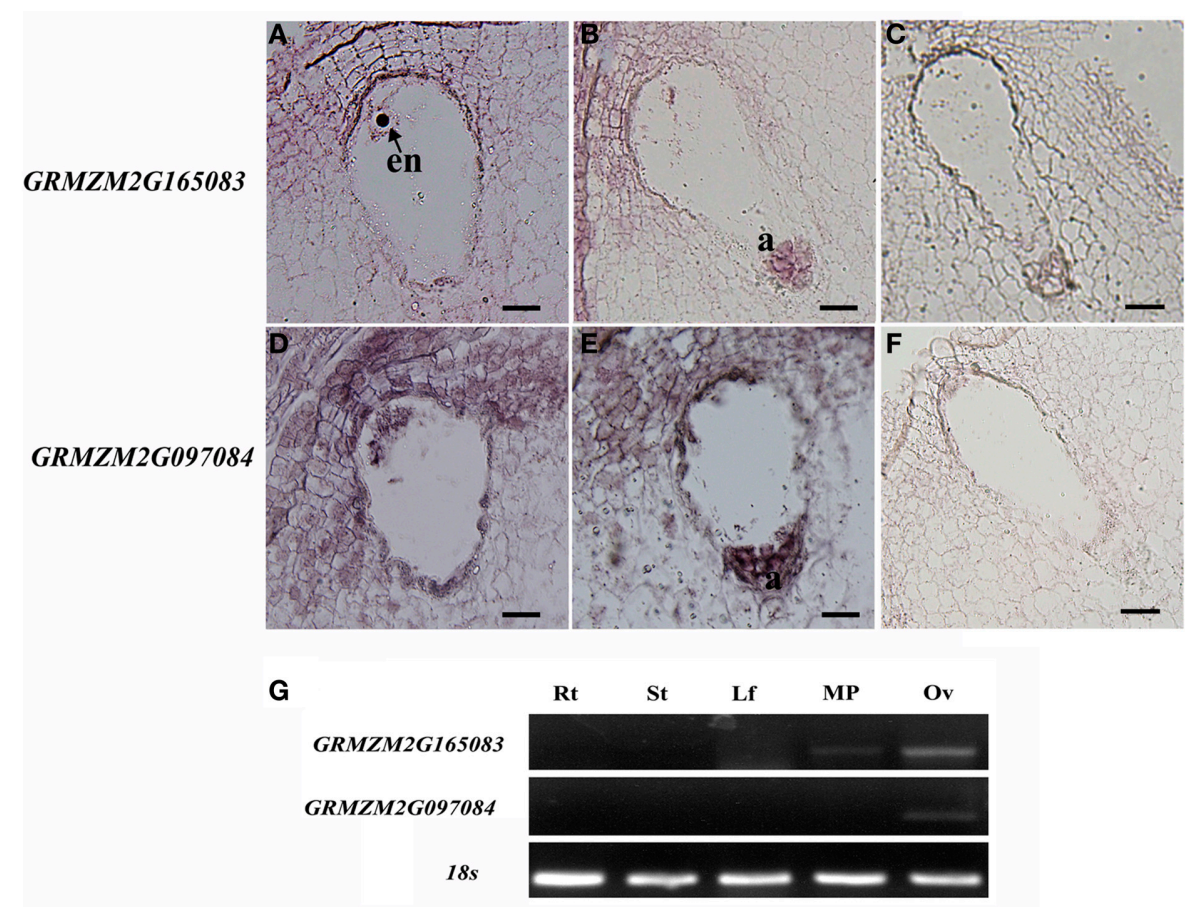

FIGURE 8 | In situ hybridization patterns of GRMZM2G165083 and GRMZM2G097084. (A,B) in situ hybridization analysis of GRMZM2G165083 expression with antisense probes; (D,E) in situ hybridization analysis of GRMZM2G097084 expression with antisense probes; (C,F) sense probes. All micrographs show longitudinal sections of maize embryo sacs. (G) Semi-quantitative RT-PCR analysis of GRMZM2G165083 and GRMZM2G097084 transcript levels in maize tissues. en, egg nucleus; a, antipodal cells; Rt, root; St, stem; Lf, leaves; MP, mature pollen; Ov, ovary. Scale bars $=20 \mu \mathrm{m}$. 


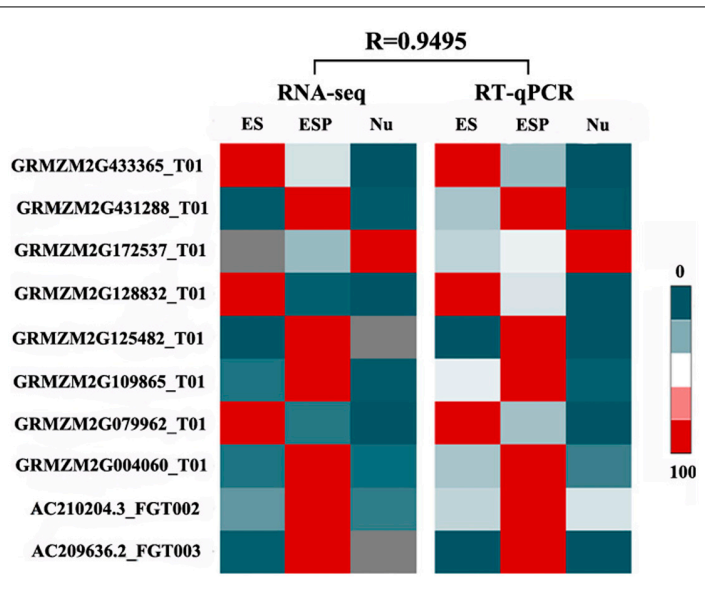

FIGURE 9 | Validation of RNA-seq results by RT-qPCR. Transcript levels of 10 randomly selected genes in the four samples were detected by RT-qPCR. A heat map (center) was constructed using mean values of transcript levels detected in three biological replicates. Normalized RPKM values of RNA-seq results are shown (left). For each gene, maximum transcript level in a certain sample was set to 100 , and transcript levels in other samples are shown relative to the maximum level. Relative transcript levels are shown by color scales (right). $\mathrm{R}$, correlation coefficient value between RNA-seq data and RT-qPCR data.

In the last decade or so, several studies have identified many molecules involved in pollen-pistil interactions and in the crosstalk between the male gametophyte and the female sporophyte. In our previous study, the transcript profiles of maize silks were analyzed at different developmental stages representing the most important events during pollination. Many genes related to microtubule-based movement, ubiquitin-mediated protein degradation, and transport were identified. These genes were involved in progamic pollen tube germination, adhesion, growth and guidance in phases I-IV (Xu et al., 2013). In phase V, speciesspecific signals or the barrier of the embryo sac itself are thought to control pollen tube growth and guidance in grasses. Thus, we conducted a preliminary analysis of transcripts involved in the embryo sac-pollen tube interaction. We identified 3467 DEGs between the ES and the ESP. Further analyses revealed that these DEGs may have roles in a number of biological pathways, including RNA binding, processing and transcription, miscellaneous enzyme family, signaling transduction, and protein targeting and degradation (Figure 3). The DEGs in the ES included genes encoding CRPs, such as ZMES4, ZMEA1, LURE1, and LURE2, and they function as key signaling factors in angiosperm reproduction (Márton et al., 2005; Dresselhaus and Márton, 2009; Okuda et al., 2009; Amien et al., 2010).

In conclusion, this RNA-seq dataset is an important resource for future study on maize reproduction. Further genetic and biochemical analysis will be critical to characterize their functions and to understand the mechanism of the maize embryo sac-pollen tube interaction.

\section{ACKNOWLEDGMENTS}

This work was supported by the Major Research Plan from the Ministry of Science and Technology of China (No.
2013CB945100) and the National Natural Science Foundation of China (No. 31100232).

\section{SUPPLEMENTARY MATERIAL}

The Supplementary Material for this article can be found online at: http://www.frontiersin.org/journal/10.3389/fpls.2014. 00702/abstract

\section{REFERENCES}

Alandete-Saez, M., Ron, M., and McCormick, S. (2008). GEX3, expressed in the male gametophyte and in the egg cell of Arabidopsis thaliana, is essential for micropylar pollen tube guidance and plays a role during early embryogenesis. Mol. Plant 1, 586-598. doi: 10.1093/mp/ssn015

Amien, S., Kliwer, I., Márton, M. L., Debener, T., Geiger, D., Becker, D., et al. (2010). Defensin-like ZmES4 mediates pollen tube burst in maize via opening of the potassium channel KZM1. PLoS Biol. 8:e1000388. doi: 10.1371/journal.pbio. 1000388

Boisson-Dernier, A., Roy, S., and Kritsas, K. (2009). Disruption of the pollen-expressed FERONIA homologs ANXUR1 and ANXUR2 triggers pollen tube discharge. Development 136, 3279-3288. doi: 10.1242/dev. 040071

Brand, U., Fletcher, J. C., Hobe, M., Meyerowitz, E. M., and Simon, R. (2000). Dependence of stem cell fate in Arabidopsis on a feedback loop regulated by CLV3 activity. Science 289, 617-619. doi: 10.1126/science.289.5479.617

Chae, K., and Lord, E. M. (2011). Pollen tube growth and guidance: roles of small, secreted proteins. Ann. Bot. 108, 627-636. doi: 10.1093/aob/mcr015

Chen, Y. H., Li, H. J., Shi, D. Q., Yuan, L., Liu, J., Sreenivasan, R., et al. (2007). The central cell plays a critical role in pollen tube guidance in Arabidopsis. Plant Cell 19, 3563-3577. doi: 10.1105/tpc.107.053967

Chettoor, A., Givan, S., Cole, R., Coker, C., Unger-Wallace, E., Vejlupkova, Z., et al. (2014). Discovery of novel transcripts and gametophytic functions via RNAseq analysis of maize gametophytic transcriptomes. Genome Bio. 15, 414. doi: 10.1186/s13059-014-0414-2

Chou, M. X., Wei, X. Y., Chen, D. S., and Zhou, J. C. (2006). Thirteen nodulespecific or nodule-enhanced genes encoding products homologous to cysteine cluster proteins or plant lipid transfer proteins are identified in Astragalus sinicus L. by suppressive subtractive hybridization. J. Exp. Bot. 57 2673-2685. doi: 10.1093/jxb/erl030

Colombo, M., Masiero, S., Vanzulli, S., Lardelli, P., Kater, M. M., and Colombo, L. (2008). AGL23, a type I MADS-box gene that controlsfemale gametophyte and embryo development in Arabidopsis. Plant J. 54, 1037-1048. doi: 10.1111/j.1365-313X.2008.03485.x

Cordts, S., Bantin, J., Wittich, P. E., Kranz, E., Lorz, H., and Dresselhaus, T. (2001). $Z m E S$ genes encode peptides with structural homology to defensins and are specifically expressed in the female gametophyte of maize. Plant J. 5, 103-114. doi: 10.1046/j.0960-7412.2000.00944.x

Covery, P. A., Subbaiah, C. C., and Parsons, R. L. (2010). A pollen-specific RALF from tomato that regulates pollen tube elongation. Plant Physiol. 153, 703-715. doi: $10.1104 /$ pp.110.155457

Dafni, A., and Motte Maues, M. (1998). A rapid and simple procedure to determine stigma receptivity. Sex. Plant Reprod. 11, 177-180. doi: 10.1007/s004970050138

Dresselhaus, T., Lausser, A., and Márton, M. L. (2011). Using maize as a model to study pollen tube growth and guidance, cross-incompatibility and sperm delivery in grasses. Ann. Bot. 108, 727-737. doi: 10.1093/aob/mcr017

Dresselhaus, T., and Márton, M. L. (2009). Micropylar pollen tube guidance and burst: adapted from defense mechanisms? Curr. Opin. Plant Biol. 12, 70-80. doi: 10.1016/j.pbi.2009.09.015

Duan, Q., Kita, D., Johnson, E. A., Aggarwal, M., Gates, L., Wu, H. M., et al. (2014). Reactive oxygen species mediate pollen tube rupture to release sperm for fertilization in Arabidopsis. Nat. Commun. 5, 3129. doi: 10.1038/ncomms4129

Dupuis, I., and Dumas, C. (1990). Biochemical markers of female receptivity in maize (Zea mays L.) assessed using in vitro fertilization. Plant Sci. 70, 11-20. doi: 10.1016/0168-9452(90)90026-K

Escobar-Restrepo, J. M., Huck, N., Kessler, S., Gagliardini, V., Gheyselinck, J., Yang, W. C., et al. (2007).The FERONIA receptor-like kinase mediates malefemale interactions during pollen tube reception. Science 317, 656-660. doi: $10.1126 /$ science. 1143562 
Guo, J., Wang, F., Song, J., Sun, W., and Zhang, X. S. (2010). The expression of Orysa;CycB1;1 is essential for endosperm formation and causes embryo enlargement in rice. Planta 231, 293-303. doi: 10.1007/s00425-009-1051-y

Heslop-Harrison, Y., Heslop-Harrison, J., and Reger, B. J. (1985). The pollenstigma interaction in the grasses. 7. Pollen-tube guidance and the regulation of tube number in Zea mays L. Acta. Bot. Neerl. 34, 193-211.

Higashiyama, T. (2002). The synergid cell: attractor and acceptor of the pollen tube for double fertilization. J. Plant Res. 115, 149-160. doi: 10.1007/s102650200020

Higashiyama, T. (2010). Peptide signaling in pollen-pistil interactions. Plant Cell Physiol. 51, 177-189. doi: 10.1093/pcp/pcq008

Higashiyama, T., and Hamamura, Y. (2008). Gametophytic pollen tube guidance. Sex Plant Reprod. 2, 17-26. doi: 10.1007/s00497-007-0064-6

Hou, X., Zhang, W., Xiao, Z., Gan, H., Lin, X., Liao, S., et al. (2012). Mining and characterization of ubiquitin E3 ligases expressed in the mouse testis. BMC Genomics 13, 495. doi: 10.1186/1471-2164-13-495

Huck, N., Moore, J. M., Federer, M., and Grossniklaus, U. (2003). The Arabidopsis mutant feronia disrupts the female gametophytic control of pollen tube reception. Development 130, 2149-2159. doi: 10.1242/dev.00458

Jiang, L., Yang, S. L., Xie, L. F., Puah, C. S., Zhang, X. Q., Yang, W. C., et al. (2005). VANGUARD1 encodes a pectin methylesterase that enhances pollen tube growth in the Arabidopsis style and transmitting tract. Plant Cell 17, 584-596. doi: 10.1105/tpc.104.027631

Johnson, M. A., and Preuss, D. (2002). Plotting a course: multiple signals guide pollen tubes to their targets. Developmental Cell 2, 273-281. doi: 10.1016/S15345807(02)00130-2

Jones-Rhoades, M. W., Borevitz, J. O., and Preuss, D. (2007). Genome-wide expression profiling of the Arabidopsis female gametophyte identifies families of small, secreted proteins. PLoS Genet. 3:e171. doi: 10.1371/journal.pgen.0030171

Kasahara, R. D., Portereiko, M. F., Sandaklie-Nikolova, L., Rabiger, D. S., and Drew, G. N. (2005). MYB98 is required for pollen tube guidance and synergid cell differentiation in Arabidopsis. Plant Cell 17, 81-92. doi: 10.1105/tpc.105.034603

Kessler, S. A., Shimosato-Asano, H., Keinath, N. F., Wuest, S. E., Ingram, G., Panstruga, R., et al. (2010). Conserved molecular components for pollen tube reception and fungal invasion. Science 330, 968-971. doi: 10.1126/science. 1195211

Kim, S., Mollet, J. C., Dong, J., Zhang, K., Park, S. Y., and Lord, E. M. (2003). Chemocyanin, a small basic protein from the lily stigma, induces pollen tube chemotropism. Proc. Natl. Acad. Sci. U.S.A. 100, 16125-16130. doi: 10.1073/pnas. 2533800100

Kim, S. T., Zhang, K. L., Dong, J., and Lord, E. M. (2006). Exogenous free ubiquitin enhances lily pollen tube adhesion to an in vitro stylar matrix and may facilitate endocytosis of SCA. Plant Physiol. 142, 1397-1411. doi: 10.1104/pp.106. 086801

Krol, E., Mentzel, T., Chinchilla, D., Boller, T., Felix, G., Kemmerling, B., et al. (2010). Perception of the Arabidopsis danger signal peptide 1 involves the pattern recognition receptor AtPEPR1 and its close homologue AtPEPR2. J. Biol. Chem. 285, 13471-13479. doi: 10.1074/jbc.M109.097394

Krusell, L., Madsen, L. H., Sato, S., Aubert, G., Genua, A., Szczyglowski, K., et al. (2002). Shoot control of root development and nodulation is mediated by a receptor-like kinase. Nature 420, 422-426. doi: 10.1038/nature01207

Lan, L., Li, M., Lai, Y., Xu, W., Kong, Z., Ying, K., et al. (2005). Microarray analysis reveals similarities and variations in genetic programs controlling pollination/fertilization and stress responses in rice (Oryza sativa L.). Plant Mol. Biol. 59, 151-164. doi: 10.1007/s11103-005-3958-4

Lausser, A., and Dresselhaus, T. (2010). Sporophytic control of pollen tube growth and guidance in grasses. Biochem. Soc. Trans. 38, 631-634. doi: 10.1042/BST0380631

Lê, Q., Gutièrrez-Marcos, J. F., Costa, L. M., Meyer, S., Dickinson, H. G., Lörz, H., et al. (2005). Construction and screening of subtracted cDNA libraries from limited populations of plant cells: a comparative analysis of gene expression between maize egg cells and central cells. Plant J. 44, 167-178. doi: 10.1111/j.1365-313X.2005.02518.x

Li, R., Yu, C., Li, Y., Lam, T. W., Yiu, S. M., Kristiansen, K., et al. (2009). SOAP2, an improved ultrafast tool for short read alignment. Bioinformatics 25, 1966-1967. doi: 10.1093/bioinformatics/btp336

Licausi, F., van Dongen, J. T., Giuntoli, B., Novi, G., Santaniello, A., Geigenberger, P., et al. (2010). HRE1 and HRE2, two hypoxia-inducible ethylene response factors, affect anaerobic responses in Arabidopsis thaliana. Plant J. 62, 302-315. doi: 10.1111/j.1365-313X.2010.04149.x
Ling, Y., Zhang, C. Y., Chen, T., Hao, H. Q., Liu, P., Bressan, R. A., et al. (2012). Mutation in SUMO E3 ligase, SIZ1, disrupts the mature female gametophyte in Arabidopsis. PLoS ONE 7:e29470. doi: 10.1371/journal.pone.0029470

Lord, E. M. (2003). Adhesion and guidance in compatible pollination. J. Exp. Bot. 54, 47-54. doi: 10.1093/jxb/erg015

Luu, D. T., Marty-Mazars, D., Trick, M., Dumas, C., and Heizmann, P. (1999). Pollen-stigma adhesion in Brassica spp involves SLG and SLR1 glycoproteins. Plant Cell 11, 251-262

Márton, M. L., Cordts, S., Broadhvest, J., and Dresselhaus, T. (2005). Micropylar pollen tube guidance by egg apparatus 1 of maize. Science 307, 573-576. doi: 10.1126/science. 1104954

Márton, M. L., and Dresselhaus, T. (2010). Female gametophyte-controlled pollen tube guidance. Biochem. Soc. Trans. 38, 627-630. doi: 10.1042/BST0380627

McInnis, S. M., Costa, L. M., Gutie'rrez-Marcos, J. F., Henderson, C. A., and Hiscock, S. J. (2005). Isolation and characterization of a polymorphic stigmaspecific class III peroxidase gene from Senecio squalidus L. (Asteraceae). Plant Mol. Biol. 57, 659-677. doi: 10.1007/s11103-005-1426-9

McInnis, S. M., Desikan, R., Hancock, J. T., and Hiscock, S. J. (2006). Production of reactive oxygen species and reactive nitrogen species by angiosperm stigmas and pollen: potential signalling crosstalk? New Phytol. 172, 221-228. doi 10.1111/j.1469-8137.2006.01875.x

Mortazavi, A., Williams, B. A., McCue, K., Schaeffer, L., and Wold, B. (2008). Mapping and quantifying mammalian transcriptomes by RNA-Seq. Nat. Methods 5, 621-628. doi: 10.1038/nmeth.1226

Mouhat, S., Jouirou, B., Mosbah, A., De Waard, M., and Sabatier, J. (2004). Diversity of folds in animal toxins acting on ion channels. Biochem. J. 378, 717-726. doi: 10.1042/BJ20031860

Muschietti, J., Dircks, L., Vancanneyt, G., and McCormick, S. (1994). LAT52 protein is essential for tomato pollen development. Plant J. 6, 321-338. doi: 10.1046/j.1365-313X.1994.06030321.x

Nakamura, N. (2013). Ubiquitination regulates the morphogenesis and function of sperm organelles. Cells 2, 732-775. doi: 10.3390/cells2040732

Nakano, T., Suzuki, K., Fujimura, T., and Shinshi, H. (2006). Genome-wide analysis of the ERF gene family in Arabidopsis and Rice. Plant Physiol. 140, 411-432. doi: 10.1104/pp.105.073783

Okuda, S., Tsutsui, H., Shiina, K., Sprunck, S., Takeuchi, H., and Yui, R. (2009). Defensin-like polypeptide LUREs are pollen tube attractants secreted from synergid cells. Nature 458, 357-361. doi: 10.1038/nature07882

Park, S. Y., and Lord, E. M. (2003). Expression studies of SCA in lily and confirmation of its role in pollen tube adhesion. Plant Mol. Boil. 51, 183-189. doi: 10.1023/A:1021139502947

Punwani, J. A., Rabiger, D. S., Lloyd, A., and Drews, G. N. (2008). The MYB98 subcircuit of the synergid gene regulatory network includes genes directly and indirectly regulated by MYB98. Plant J. 55, 406-414. doi: 10.1111/j.1365313X.2008.03514.x

Robinson, M. D., McCarthy, D. J., and Smyth, G. K. (2010). edgeR, a Bioconductor package for differential expression analysis of digital gene expression data. Bioinformatics 26, 139-140. doi: 10.1093/bioinformatics/btp616

Rotman, N., Rozier, F., Boavida, L., Dumas, C., and Berger, F. (2003). Female control of male gamete delivery during fertilization in Arabidopsis thaliana. Curr. Biol. 13, 432-436. doi: 10.1016/S0960-9822(03)00093-9

Sanchez, A. M., Bosch, M., Bots, M., Nieuwland, J., Feron, R., and Mariani, C. (2004). Pistil factors controlling pollination. Plant Cell 16, S98-S106. doi: 10.1105/tpc.017806

Schnabel, E., Journet, E. P., de Carvalho-Niebel, F., Duc, G., and Frugoli, J. (2005). The Medicago truncatula SUNN gene encodes a CLV1-like leucine-rich repeat receptor kinase that regulates nodule number and root length. Plant Mol. Biol. 58, 809-822. doi: 10.1007/s11103-005-8102-y

Sharoni, A. M., Nuruzzaman, M., Satoh, K., Shimizu, T., Kondoh, H., Sasaya, T., et al. (2011). Gene structures, classification and expression models of the AP2/EREBP transcription factor family in rice. Plant Cell Physiol. 52, 344-360. doi: $10.1093 / \mathrm{pcp} / \mathrm{pcq} 196$

Shiu, S. H., Karlowski, W. M., Pan, R., Tzeng, Y. H., Mayer, K. F., and Li, W. H. (2004). Comparative analysis of the receptor-like kinase family in Arabidopsis and rice. Plant Cell 16: 1220-1234. doi: 10.1105/tpc.020834

Silverstein, K. A., Moskal, W. A. Jr., Wu, H. C., Underwood, B. A., Graham, M. A., Town, C. D., et al. (2007). Small cysteine-rich peptides resembling antimicrobial peptides have been under-predicted in plants. Plant J. 51, 262-280. doi: 10.1111/j.1365-313X.2007.03136.x 
Singhmb, X., Bhallapl, L., Zhang, Z., Swobodai, M., and Russells, D. (2002). Developmental expression of polyubiquitin genes and distribution of ubiquitinated proteins in gene rative and sperm cells. Sex Plant Reprod. 14, 325-329. doi: 10.1007/s00497-001-0126-0

Sprunck, S., Baumann, U., Edwards, K., Langridge, P., and Dresselhaus, T. (2005). The transcript composition of egg cells changes significantly following fertilization in wheat (Triticum aestivum L.). Plant J. 41, 660-672. doi: 10.1111/j.1365313X.2005.02332.x

Sutovsky, P., Moreno, R., Ramalho-Santos, J., Dominko, T., Thompson, W. E., and Schatten, G. (2001). A putative, ubiquitin-dependent mechanism for the recognition and elimination of defective spermatozoa in the mammalian epididymis. J. Cell Sci. 114, 1665-1675.

Takayama, S., Shiba, H., Iwano, M., Asano, K., Hara, M., Che, F. S., et al. (2000). Isolation and characterization of pollen coat proteins of Brassica campestris that interact with S locus-related glycoprotein 1 involved in pollen-stigma adhesion. Proc. Natl. Acad. Sci. U.S.A. 97, 3765-3770. doi: 10.1073/pnas.97.7.3765

Wuest, S. E., Vijverberg, K., Schmidt, A., Weiss, M., Gheyselinck, J., Lohr, M., et al. (2010). Arabidopsis female gametophyte gene expression map reveals similarities between plant and animal gametes. Curr. Biol. 20, 506-512. doi: 10.1016/j.cub.2010.01.051

Xu, X. H., Chen, H., Sang, Y. L., Wang, F., Ma, J. P., and Zhang, X. S. (2012). Identification of genes specifically or preferentially expressed in maize silk reveals similarity and diversity in transcript abundance of different dry stigmas. BMC Genomics 13:294. doi: 10.1186/1471-2164-13-294

Xu, X. H., Wang, F., Chen, H., Sun, W., and Zhang, X. S. (2013). Transcript profile analyses of maize silks reveal effective activation of genes involved in microtubule-based movement, ubiquitin-dependent protein degradation, and transport in the pollination process. PLoS ONE 8:e53545. doi: 10.1371/journal. pone. 0053545

Yamaguchi, Y., Huffaker, A., Bryan, A. C., Tax, F. E., and Ryan, C. A. (2010). PEPR2 is a second receptor for the Pep1 and Pep2 peptides and contributes to defense responses in Arabidopsis. Plant Cell 22, 508-522. doi: 10.1105/tpc.109. 068874
Yamaguchi, Y., Pearce, G., and Ryan, C. A. (2006). The cell surface leucine-rich repeat receptor for AtPep1, an endogenous peptide elicitor in Arabidopsis, is functional in transgenic tobacco cells. Proc. Natl. Acad. Sci. U.S.A. 103, 10104-10109. doi: 10.1073/pnas.0603729103

Yang, H., Kaur, N., Kiriakopolos, S., and McCormick, S. (2006). EST generation and analyses toward identifying female gametophyte-specific genes in Zea mays L. Planta 224, 1004-1014. doi: 10.1007/s00425-0060283-3

Yu, H. J., Hogan, P., and Sundaresan, V. (2005). Analysis of the female gametophyte transcriptome of arabidopsis by comparative expression profiling. Plant Physiol. 139, 1853-1869. doi: 10.1104/pp.105.067314

Zhang, X. S., and O'Neill, S. D. (1993). Ovary and gametophyte development are coordinately regulated by auxin and ethylene following pollination. Plant Cell 5, 403-418.

Conflict of Interest Statement: The authors declare that the research was conducted in the absence of any commercial or financial relationships that could be construed as a potential conflict of interest.

Received: 29 September 2014; accepted: 25 November 2014; published online: 17 December 2014.

Citation: Wang SS, Wang F, Tan SJ, Wang MX, Sui N and Zhang XS (2014) Transcript profiles of maize embryo sacs and preliminary identification of genes involved in the embryo sac-pollen tube interaction. Front. Plant Sci. 5:702. doi: 10.3389/fpls. 2014.00702

This article was submitted to Plant Genetics and Genomics, a section of the journal Frontiers in Plant Science.

Copyright (c) 2014 Wang, Wang, Tan, Wang, Sui and Zhang. This is an open-access article distributed under the terms of the Creative Commons Attribution License (CC BY). The use, distribution or reproduction in other forums is permitted, provided the original author(s) or licensor are credited and that the original publication in this journal is cited, in accordance with accepted academic practice. No use, distribution or reproduction is permitted which does not comply with these terms. 